\title{
The Collective Intelligence based Program to Accelerate Achievement of the Sustainable Development Goals as a Case Study for Collectively Intelligent Program Design
}

\author{
Abstract \\ General Collective Intelligence (GCI) are software platforms that organize groups into a single \\ collective intelligence with general problem solving ability. In doing so a GCI has the potential to give \\ groups vastly more ability to address collective challenges such as the SDGs. A GCI is a significant \\ infrastructure investment. The Collective Intelligence based Program to Accelerate Achievement of the \\ Sustainable Development Goals (CIPAA-SDGs) however is designed to implement a GCI in phases so \\ that the cost to any single project is far outweighed by the potential benefits. The phasing of that GCI \\ development in the CIPAA-SDGs program design is used here as a case study for collectively \\ intelligent program design.
}

\section{Introduction}

The Collective Intelligence based Program to Accelerate Achievement of the Sustainable Development Goals (CIPAA-SDGs) is a case study for program design that aims to incorporate GCI to scale capacity for problem solving, and to scale capacity cooperation to scale capacity for impact while also minimizing the issue of solving the wrong problem [1],[4]. Doing so requires facilitating a bottom-up approach allowing any participant to introduce solutions such as modes of cooperation, thereby increasing the capacity to benefit from a wider range of cooperative strategies. In a bottom up approach any individual might suggest such a strategy, and rather than a single decision-maker deciding which is adopted, a collective intelligence process within the GCI would orchestrates the group in collectively adopting the choice that optimizes collective outcomes. Other processes within the GCI would then collectively evolve the chosen solution, and eventually learn, and use collective reasoning to improve it. The goal of applying GCI is to give groups vastly greater capacity to address collective problems through much more powerful social impact programs.

The design of the CIPAA-SDGs explores how a program can be designed to systematically implement several dimensions of cooperation that are likely impossible in current international development programs. As an example of one dimension of cooperation, consider a program to provide rural communities with water. It might be true that a new much more effective and cost efficient water pump can be designed. And it might be true that technologies enabling even greater effectiveness and efficiency can be researched. But people have urgent water needs today. How much resources should be invested in deploying the water pumps available today, how much invested in research, development, and how much planned for implementation of the new pumps?

The aim of collective intelligence is to turn this question into a maximization problem that can be approached mathematically, so the group can cooperate through a methodical process to answer it. 
Allocate Resources Across Implementation, Development, and Research to Maximize Impact

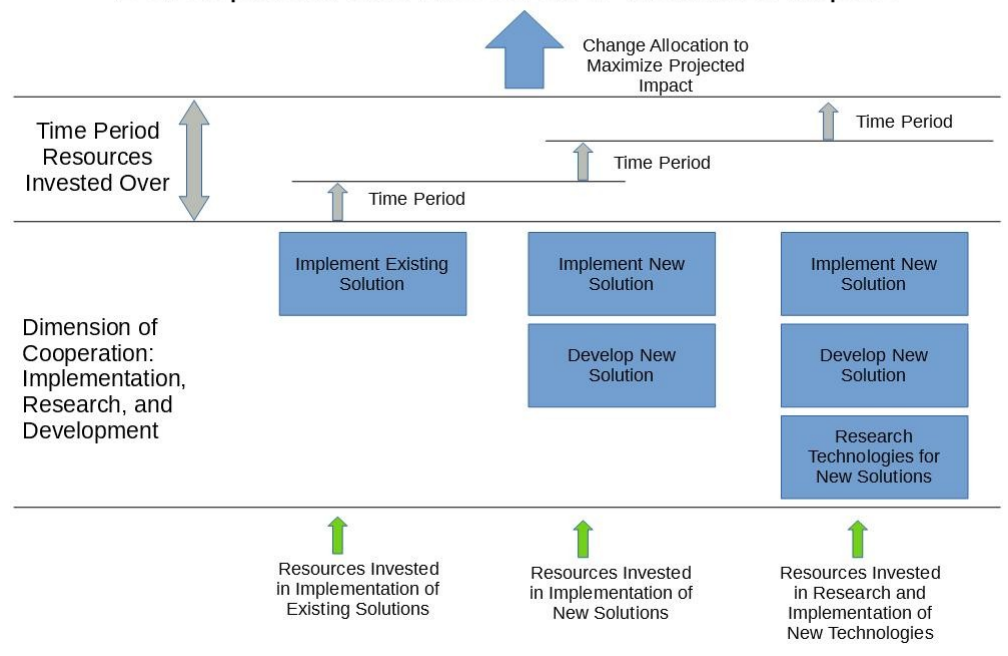

This is an instance of a general pattern in which the program maximizes impact within a given dimension of cooperation.

General Pattern: Maximize Impact by Allocating Resources Across Different Scopes of Cooperation for each Dimension of Cooperation

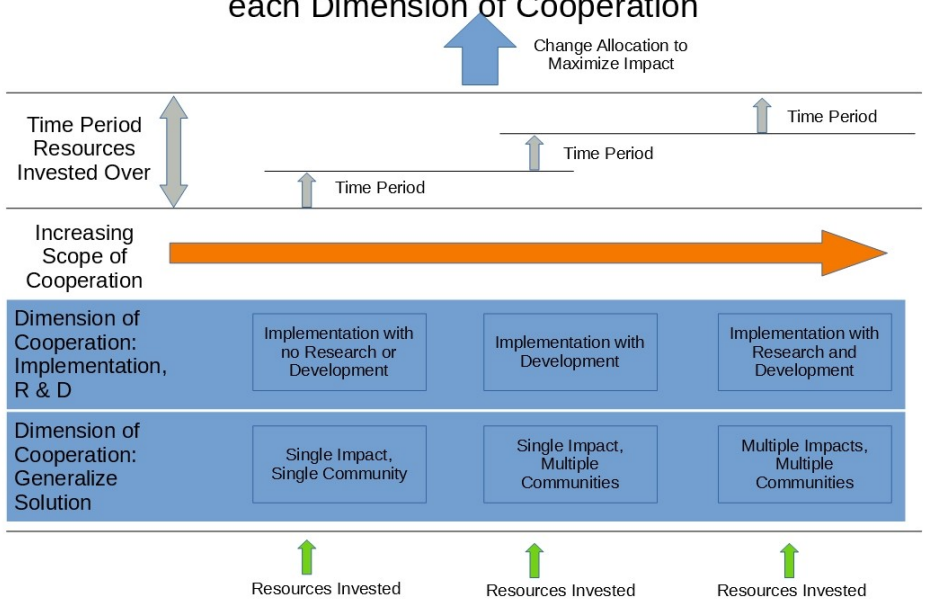

Within collectively intelligent programs this pattern is reused to maximize impact across all dimensions of cooperation (all regions, program phases, timescales, etc.), simultaneously. 
Allocate Resources Globally to Maximize Impact

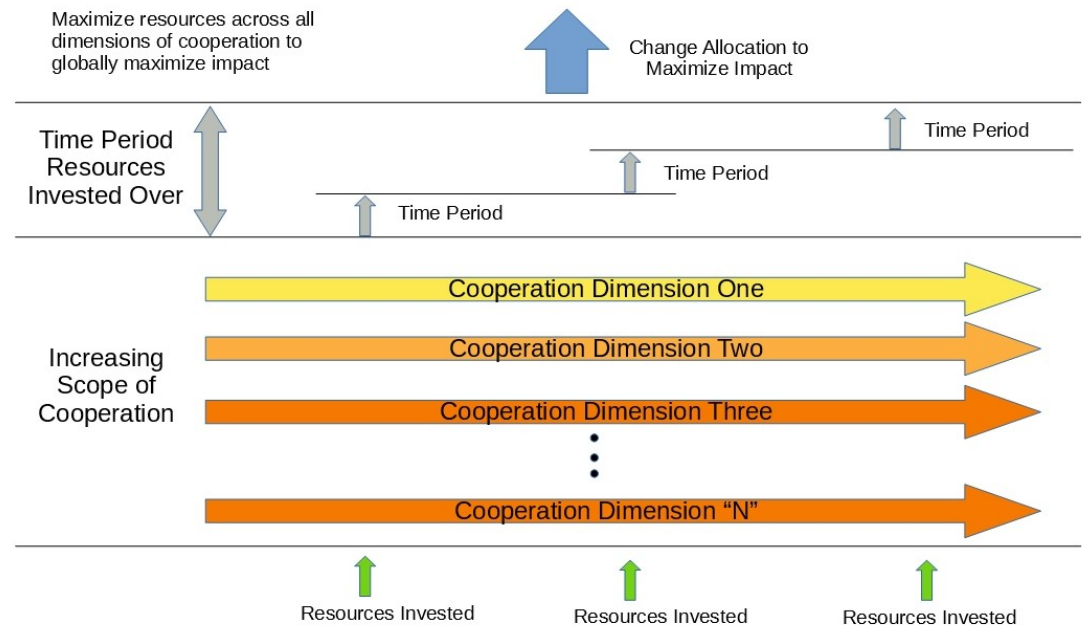

\section{Phase I of the CIPAA-SDGs Initiative: Program to Improve Farmer Livelihoods by Increasing Demand for Local Agricultural Production}

Phase I of the proposed CIPAA-SDGs Initiative aims to launch an Agricultural Livelihoods program in each of the target regions. The key attribute of cooperation in this phase is that it is limited to cooperating with governments donors and others to deploy entire value chains of local businesses that cooperate increase impact on agricultural livelihoods, and that these value chains require no significant development of new products or service. This initiative is proposed to be launched in two regions. One is proposed to be the four east African countries (Kenya, Rwanda, Uganda, Tanzania). And the second is proposed to be the fifteen Caribbean Community (CARICOM) nations.

Phase I of this program in the pilot country proposes to direct $\$ 30$ million USD in external funding to improve farmer livelihoods by increasing demand for local agricultural produce. For phase II of this program a key attribute of cooperation is the development of new products and services with the potential to significantly increase impact. In phase II the agriculture program will direct a far larger investment towards development of advanced hydroponic and aquaponic systems that will allow the phase II pilot nation to grow a wide range of produce it currently imports, where the value of implementing these advanced agricultural systems across the entire CARICOM region will justify a much larger investment in the development of those systems than could be warranted by the size of the market in the pilot country alone.

Rather than fund the creation of individual businesses, this collective intelligence approach funds entire value chains of businesses that cooperate to become sustainably self-supporting [2]. In the CARICOM nations, the pilot of this program proposes to deploy a \$2 million USD value chain of local businesses in each CARICOM member state, where this value chain can be replicated to all fifteen CARICOM countries. The cooperative synergies of the program create the potential for well above market returns for investors, so that with the proven success of the value chains in the pilot nations, investors are incentivized to replicate the value chains in all other CARICOM member states as well. 
The instrument of cooperation that will be used to incentivize participation in these value chains is called the "impact bond". The impact bond is a commitment from a donor or government to provide a grant paid in terms of cash, or in terms of tax credits, to compensate a business in the value chain for achieving a specific amount of impact, such as job creation. Through these impact bonds governments or donors can significantly increase their impact per dollar spent (up to 50X as in Appendix 1:

Proposed Agricultural Value Chain in Antigua and Barbuda). And the increased profits created by these impact bonds create the possibility of investment returns that are significantly above market averages, to reliably incentivize private investment in those value chains.

The result is that the program projects a total of $\$ 30$ million USD in job creation investment can reliably be achieved in each CARICOM member state. Because these value chains can be replicated in the other CARICOM nations as well, the total economic impact across the CARICOM region may be up to $\$ 450$ million USD.

\section{Healthcare Section of Phase II of the CIPAA-SDGs Initiative: Program to Improve Healthcare Outcomes}

Phase II of the proposed CIPAA-SDGs Initiative aims to launch an Affordable Access to Healthcare program, an Affordable Access to Education Program, and Affordable Access to Renewable Energy Program, and other programs. The key attribute of cooperation in this phase is that cooperation will also be used to develop new products or services with the potential to significantly increase impact.

The healthcare section of this program will develop two healthcare solutions in the pilot country, where those solutions leverage the principles of collective intelligence to more efficiently orchestrate cooperation between healthcare providers, insurers, patients, and others. Through this cooperation, these solutions are projected to create the capacity to achieve significant healthcare savings in the deployment region, or a significant increase in the services that can be delivered at the same cost, and thereby to increase access to affordable healthcare across the region.

These healthcare solutions proposed for phase II of the CIPAA-SDGs are the Provider Based Health Insurance Solution, and the National Electronic Health Record (EHR)/Single Virtual Patient Record Solution [3].

The CIPAA-SDGs program uses collective intelligence to systematically build large-scale cooperation with the potential for transformative impact on access to affordable healthcare. And through this cooperation this program creates the opportunity to leverage the projected cost savings across the entire deployment region to incentivize a far larger investment in developing these solutions than would be justified by the size of any single deployment country alone.

This incentive is provided through impact capacity bonds that commit other countries to reimburse this program's investment in solution development with a portion of the savings they actually achieve with a solution that provides the capacity for this impact on cost saving. For example, if even one fifth of the potential 50\% cost savings are reliably possible, an impact capacity bond from another CARICOM member state that committed to reimburse this program with $5 \%$ of the savings achieved by them, over all of CARICOM could amount to over 50 times the investment in development of these solutions than could be justified by the size of the healthcare market in the pilot country alone. Through this cooperation, this program aims to create solutions that address specific healthcare challenges shared by all the islands, that aren't solvable within the resources of any island alone.

\section{General Collective Functionality Built Per Phase}


The collective intelligence platforms to be developed in each of the first few phases of the CIPAASDGs program are listed below along with the GCI functionality those platforms implement. Dictating the focus of each phase of the program in this top-down centralized way is necessary at the beginning. Later phases of the CIPAA-SDGs program are not listed here. But those steps are expected to be refined as the ability to do so in decentralized collective process is implemented over the first few phases.

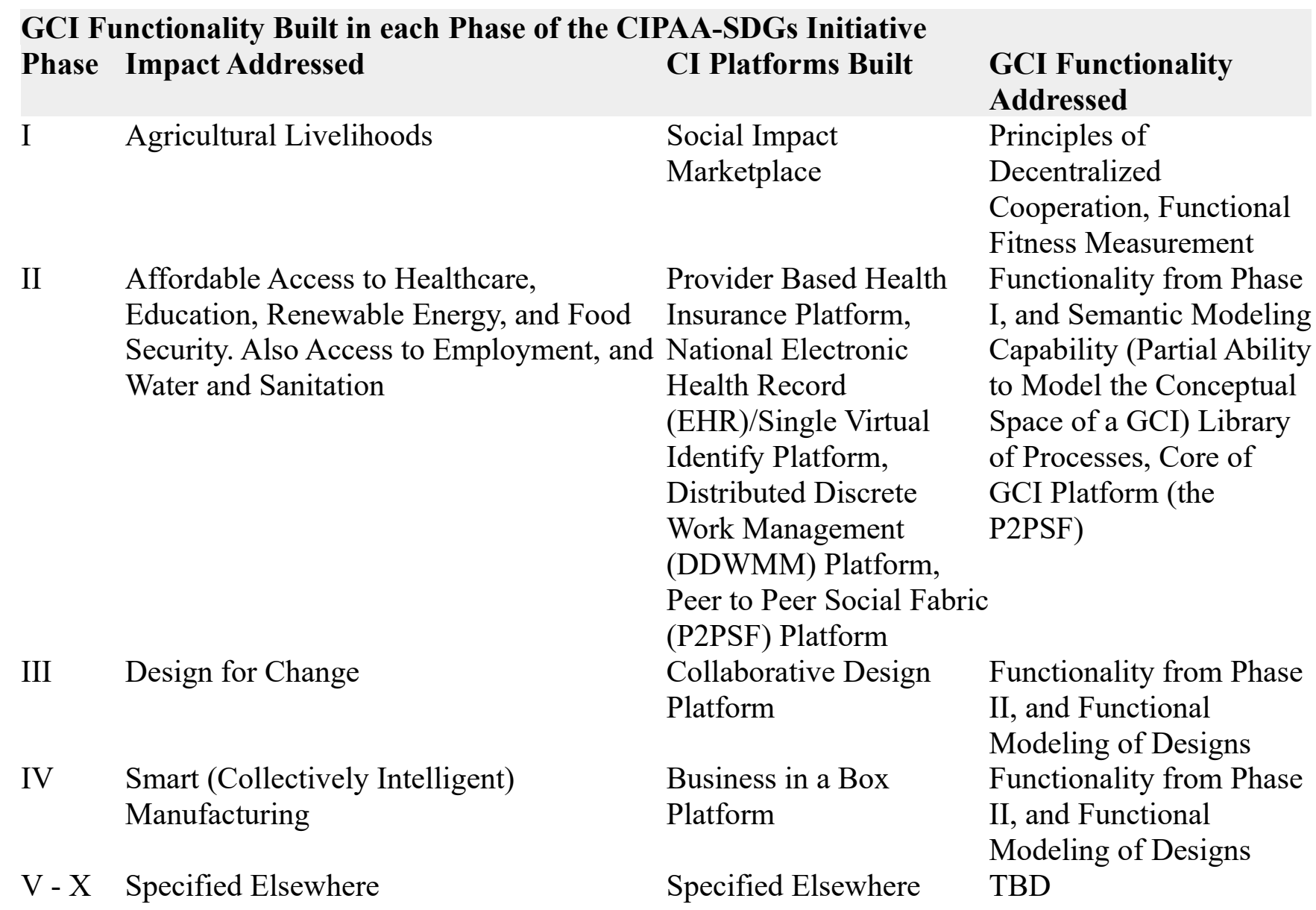

Conclusions

Even defining an initiative with a series of such massive programs is extremely ambitious, but if it is true that a system of GCI is required to have the ability to reliably address the SDGs, and if it is true that a GCI has the potential to make global achievement of the SDGs self-funding once launched so that addressing the $\$ 23$ trillon SDGs funding gap is reliably possible, then developing a system of GCI is critical. Coordinating its development across a program such as the CIPAA-SDGs, and thereby splitting the cost over the phases of such a program, is the only way to make that development affordable.

\section{References}

[1] Andy E. Williams, The Need for General Collective Intelligence in Addressing the Sustainable Development Goals, DOI:10.21203/rs.3.rs-18022/v1.

[2] Andy E. Williams, The Design of the Social Impact Marketplace as a Partial Implementation of a General Collective Intelligence (GCI) Platform, working paper 
[3] Andy E. Williams, A Conceptural Provider Based Insurance Platform and a Conceptual National Electronic Health Record (EHR)/ Single Virtual Patient Identity Platform as Partial Implemenations of a General Collective Intelligence (GCI) Platform, working paper

[4] Williams, A. E. (2020, April 16). General Collective Intelligence and the Constraints to Group Decision-Making. https://doi.org/10.31234/osf.io/6gtcn 